\title{
Polychromatic cone-beam phase-contrast tomography
}

\author{
G. R. Myers, ${ }^{1, *}$ S. C. Mayo, ${ }^{2}$ T. E. Gureyev ${ }^{2}$ D. M. Paganin,${ }^{1}$ and S. W. Wilkins ${ }^{2}$ \\ ${ }^{1}$ School of Physics, Monash University, VIC 3800, Australia \\ ${ }^{2}$ CSIRO Manufacturing and Materials Technology, PB 33, Clayton South, VIC 3169, Australia
}

(Received 5 January 2007; revised manuscript received 8 May 2007; published 24 October 2007)

\begin{abstract}
A method is presented for quantitative phase-contrast tomography using unfiltered radiation from a small polychromatic source. The three-dimensional distribution of complex refractive index in a monomorphous object is reconstructed given a single projection image per view angle. The reconstruction algorithm is achromatic and stable with respect to high-spatial-frequency noise, in contrast to conventional tomography. The density distribution in a test sample was accurately reconstructed from polychromatic phase-contrast data collected with a point-projection x-ray microscope.
\end{abstract}

DOI: 10.1103/PhysRevA.76.045804

PACS number(s): 42.30.Wb, 42.30.Rx, 81.70.Tx, 87.59.Fm

Conventional absorption-based computed tomography (CT) is a method of choice for three-dimensional imaging $[1,2]$, differentiating regions within the sample based on their attenuation of penetrating radiation or matter waves ( $\mathrm{x}$ rays, neutrons, electrons, etc.). Consequently, features displaying weak absorption contrast-such as different soft tissues in the case of medical $x$ rays, or different transparent materials in neutron radiography - are difficult to resolve using conventional CT $[3,4]$. However, if one has knowledge of not only the intensity but also the phase of the transmitted wave for a number of viewing angles (projections) about the object, one may be able to reconstruct its three-dimensional distribution of complex refractive index. This enables the differentiation of features on the basis of their refractive properties, which are otherwise invisible to conventional absorption-based CT. The use of refraction, rather than absorption, as a contrast mechanism has an important advantage in the possibility for a significant reduction of the absorbed radiation dose required for achieving an acceptable signal-to-noise ratio in the images. Furthermore, exploiting phase contrast and reducing absorption, e.g., by using highenergy polychromatic beams, one can minimize beam hardening artifacts [1] which are known to negatively affect the quantitative accuracy of conventional CT reconstruction. Of course, it is not feasible to measure wave-field phase directly, at optical and higher frequencies. Instead, in phase-contrast CT (PCT) one may employ appropriate phase-retrieval techniques to determine the object-plane phase, either explicitly or implicitly, from intensity measurements [2,5-21].

In this Brief Report we focus on PCT techniques in which a weakly absorbing object is illuminated by divergent partially coherent paraxial waves emanating from a source traversing a circular path around the object, the reconstruction of the three-dimensional complex refractive index distribution being achieved on the basis of the phase contrast which occurs as a result of free-space propagation of transmitted waves from the object to the detector [3,22]. Methods for propagation-based PCT have been subject to much active research in recent years $[5,6,9-11,14-16,18,21]$. However, in most of the published works monochromatic incident waves

\footnotetext{
*glenn.myers@sci.monash.edu.au
}

were assumed. While well suited to, e.g., typical synchrotron-based experiments, such a methodology cannot be applied directly to PCT with conventional sources, which typically generate beams that are both polychromatic and divergent $[14,19]$. In the latter case the monochromatization and collimation of incident radiation is usually impractical as it strongly reduces the incident flux and, consequently, increases the exposure times beyond acceptable limits. Given that propagation-based phase contrast may be exhibited using unfiltered radiation from polychromatic sources of sufficiently small diameter [22], it is timely that the effects of polychromaticity, partial spatial coherence, and divergence be explicitly incorporated into PCT reconstruction algorithms. Remarkably, as shown below, in the special case of nonabsorbing objects and sufficiently short propagation distances between the object and the detector, the propagationbased PCT is achromatic, thus allowing quantitative conebeam PCT to be realized using unfiltered divergent radiation from laboratory-based x-ray microfocus sources, as well as small polyenergetic sources of cold or thermal neutrons. The method may also be applied to point-projection optical tomography using a thermal light source, and to the minimally destructive three-dimensional imaging of cold atom clouds [23].

The method of polychromatic PCT can also be extended to weakly absorbing "monomorphous" (i.e., "homogeneous" [24]) objects provided the wavelength spectrum is not too broad (see details below). Recall that an object is called monomorphous with respect to the incident radiation if the distribution of its complex refractive index $n(\mathbf{r}, \lambda)=1-\Delta(\mathbf{r}, \lambda)+i \beta(\mathbf{r}, \lambda)$ is such that the ratio $\varepsilon(\lambda)$ $=\beta(\mathbf{r}, \lambda) / \Delta(\mathbf{r}, \lambda)$ is independent of position $\mathbf{r}$, at all wavelengths present in the detected spectrum. The assumption of monomorphicity is valid for $\mathrm{x}$-ray-illuminated or neutronilluminated objects composed of a single material $[14,24]$, for objects composed of light elements $(Z<10)$ illuminated by high energy $(60-500 \mathrm{keV}) \mathrm{x}$ rays [16], and also for cold clouds of two-level atoms illuminated with off-resonant light $[23,25]$. Note that nonabsorbing objects constitute a subset of monomorphous objects, with $\varepsilon(\lambda) \equiv 0$ for all incident wavelengths. For weakly absorbing monomorphous objects, $\varepsilon(\lambda)$ is assumed small.

Let the object lie entirely within a sphere of radius $d$ 


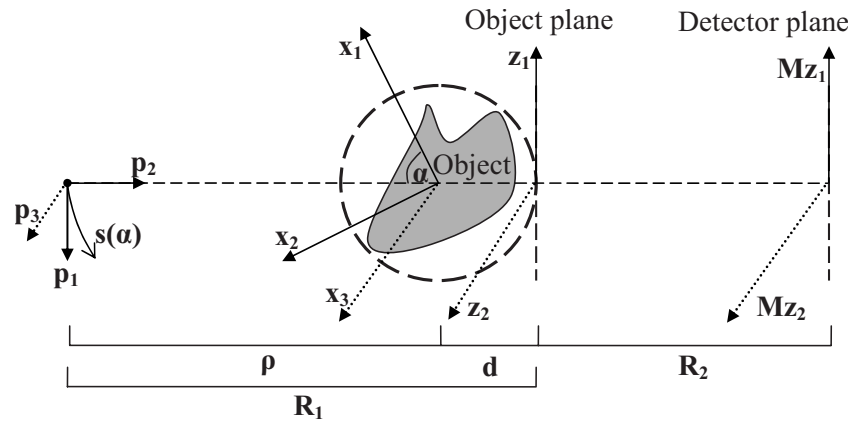

FIG. 1. Schematic of experimental layout for polychromatic cone-beam phase-contrast tomography.

centered at the origin of the Cartesian coordinates $\mathbf{x}$ $=\left(x_{1}, x_{2}, x_{3}\right)$. Consider first a point source located at position $\mathbf{s}(\alpha)=(\rho \cos \alpha, \rho \sin \alpha, 0)$, emitting radiation with wave number $k=2 \pi / \lambda$. This source traces a circle of radius $\rho$ in the $x_{1}-x_{2}$ plane centered at $\mathbf{x}=0$, where $\alpha \in[0,2 \pi)$ is the angle from the $x_{1}$ axis to the source (see Fig. 1). The distance from the source to the object plane is $R_{1}=\rho+d$. Assuming the projection approximation [21] is valid within the object, the object-plane phase $\phi_{0}(\mathbf{z}, \alpha, \lambda)$ of a monochromatic component of the illuminating beam is given by the line integral

$$
\phi_{0}(\mathbf{z}, \alpha, \lambda)=-k \int_{-\infty}^{\infty} \Delta\left(\mathbf{s}(\alpha)+\frac{\mathbf{p}}{|\mathbf{p}|} t, \lambda\right) d t,
$$

where $\mathbf{z}=\left(z_{1}, z_{2}\right)=\left(-R_{1} p_{1} / p_{2}, R_{1} p_{3} / p_{2}\right)$ is a Cartesian coordinate system in the object plane (see Fig. 1), and $\mathbf{p}=\left(p_{1}, p_{2}, p_{3}\right)=\left(x_{2} \cos \alpha-x_{1} \sin \alpha, \rho-x_{1} \cos \alpha-x_{2} \sin \alpha, x_{3}\right)$ is a Cartesian coordinate system rotated by an angle $\pi / 2+\alpha$ about the $x_{3}$ axis with respect to $x_{1}$, that is centered on the x-ray source (see Fig. 1). Given knowledge of the object-plane phase for projections $\alpha \in[0,2 \pi)$, we can reconstruct the real refractive-index decrement $\Delta(\mathbf{x}, \lambda)$ using the well-known Feldkamp-Davis-Kress (FDK) reconstruction algorithm $[26,27]$ :

$\Delta(\mathbf{x}, \lambda)=-\frac{R_{1}^{2} \rho}{2 k} \int_{0}^{2 \pi} \frac{1}{p_{2}^{2}} \mathbf{F}_{1}^{-1}\left[\left|\xi_{1}\right| \mathbf{F}_{1}\left[\frac{\phi_{0}(\mathbf{z}, \alpha, \lambda)}{\sqrt{R_{1}^{2}+z_{1}^{2}+z_{2}^{2}}}\right]\right] d \alpha$,

where $\mathbf{F}_{1}$ is the one-dimensional Fourier transform with $\xi_{1}$ dual to $z_{1}$.

To render the phase of the object-plane wave field visible as intensity variations in a propagation-based phase-contrast image, a propagation distance $R_{2}>0$ is introduced between the object plane and the detector (see Fig. 1). It is assumed that the transmitted wave is paraxial, and that the Guigay condition [28], $\left|\phi_{0}(\mathbf{z}, \alpha, \lambda)-\phi_{0}\left(\mathbf{z}+R^{\prime} \lambda \boldsymbol{\xi}, \alpha, \lambda\right)\right|<<1$, holds for any point $\mathbf{z}$ in the object plane and any $\xi$ from the support of the spatial Fourier spectrum of the transmitted wave, $\boldsymbol{\xi}=\left(\xi_{1}, \xi_{2}\right)$ being dual to $\mathbf{z}=\left(z_{1}, z_{2}\right)$, where $R^{\prime}=R_{2} / M$, $M=\left(R_{1}+R_{2}\right) / R_{1}$ is the geometric magnification, and $\xi \equiv|\xi| \leqslant 1 /\left(2 h_{\min }\right)$, where $h_{\min }$ is the size of the minimal resolvable feature in the image. Then the free-space propagation of the transmitted wave can be described by the following linear expression:

$$
M^{2} S_{R_{2}}(M \mathbf{z}, \alpha, \lambda)=S_{\text {in }}(\lambda)\left\{1+2 \mathbf{F}_{2}^{-1}\left[T(\xi, \lambda) \mathbf{F}_{2}\left[\phi_{0}(\mathbf{z}, \alpha, \lambda)\right]\right]\right\},
$$

where $S_{R_{2}}(M \mathbf{z}, \alpha, \lambda)$ is the spectral density at the detector plane, $S_{i n}(\lambda)$ is the unperturbed spectral density in the object plane, $\mathbf{F}_{2}$ is the two-dimensional Fourier transform, and $T(\boldsymbol{\xi}, \lambda)=\sin \left(\pi \lambda R^{\prime} \xi^{2}\right)+\varepsilon(\lambda) \cos \left(\pi \lambda R^{\prime} \xi^{2}\right) \quad$ is the phasecontrast transfer function in the case of a weakly absorbing monomorphous object $[23,29]$.

The paraxial approximation made in deriving Eq. (3) is equivalent to assuming a small cone-beam angle and consequently neglecting terms of second or higher order in $d / R_{1}$. Applying this approximation to Eq. (2), subsequently making use of both Eq. (3) and the identity $\left|\xi_{1}\right| \mathbf{F}_{2}[1] \equiv 0$, the former equation becomes

$$
\begin{aligned}
\Delta(\mathbf{x}, \lambda)= & -\frac{R_{1} \rho M^{2}}{4 k S_{i n}(\lambda)} \int_{0}^{2 \pi} \frac{1}{p_{2}^{2}} \mathbf{F}_{2}^{-1} \\
& \times\left[\frac{\left|\xi_{1}\right|}{T(\xi, \lambda)} \mathbf{F}_{2}\left[S_{R_{2}}(M \mathbf{z}, \alpha, \lambda)\right]\right] d \alpha .
\end{aligned}
$$

Thus we have derived a paraxial cone-beam phase-andamplitude computed tomography (PACT) reconstruction formula which is valid for a monochromatic point source illuminating a weakly absorbing monomorphous object. It allows one to reconstruct the complex refractive index $n(\mathbf{x}, \lambda)=1+\Delta(\mathbf{x}, \lambda)[i \varepsilon(\lambda)-1]$ of an object from a single phase-contrast image taken at each projection angle $\alpha \in[0,2 \pi)$. The PACT algorithm for plane incident waves [18] is a special case of Eq. (4), corresponding to the limit $\rho \rightarrow \infty$.

The above result may be generalized to the case of a polychromatic source producing an incident spectral density distribution $S_{i n}(\lambda)$ in the object plane. Let us first consider nonabsorbing objects $[\varepsilon(\lambda) \equiv 0]$, and also assume that the propagation distance satisfies the "near-field" condition, $R^{\prime} \ll h_{\min }^{2} / \lambda$. As usual, the latter condition allows one to apply the following approximation: $T(\xi, \lambda) \equiv \sin \left(\pi \lambda R^{\prime} \xi^{2}\right)$ $\cong \pi \lambda R^{\prime} \xi^{2}$, and hence $k T(\xi, \lambda) \cong 2 \pi^{2} R^{\prime} \xi^{2}$. Note that this second-order Taylor approximation, to the transcendental transfer function $T(\xi, \lambda)$, may also be obtained via an analysis based on the so-called transport-of-intensity equation [21]. This second-order expression for $k T(\xi, \lambda)$ is quite remarkable in that it does not depend on $\lambda$. If we now multiply both sides of Eq. (4) by $S_{i n}(\lambda) D(\lambda), D(\lambda)$ being the spectral sensitivity of the detector, integrate the result over the wavelength spectrum, and then divide both sides by the polychromatic (time-averaged) incident intensity, $I_{\text {in }}=\int_{0}^{\infty} S_{\text {in }}(\lambda) D(\lambda) d \lambda$, we obtain

$$
\Delta_{S}(\mathbf{x})=-\frac{\rho M^{3}}{8 \pi^{2}(M-1) I_{\text {in }}} \int_{0}^{2 \pi} \frac{1}{p_{2}^{2}} \mathbf{F}_{2}^{-1}\left[\frac{\left|\xi_{1}\right| \mathbf{F}_{2}\left[I_{R_{2}}(M \mathbf{z}, \alpha)\right]}{\xi^{2} \mathbf{F}_{2}\left[P_{M}(M \mathbf{z})\right]}\right] d \alpha,
$$

where $\Delta_{S}(\mathbf{x})=\int_{0}^{\infty} S_{i n}(\lambda) D(\lambda) \Delta(\mathbf{x}, \lambda) d \lambda / I_{\text {in }}$ is the spectrally averaged decrement of the real refractive index, $I_{R_{2}}(\mathbf{z}, \alpha)$ $=P_{M}(\mathbf{z}) * \int_{0}^{\infty} S_{R_{2}}(\mathbf{z}, \alpha, \lambda) D(\lambda) d \lambda$ is the detected polychromatic 


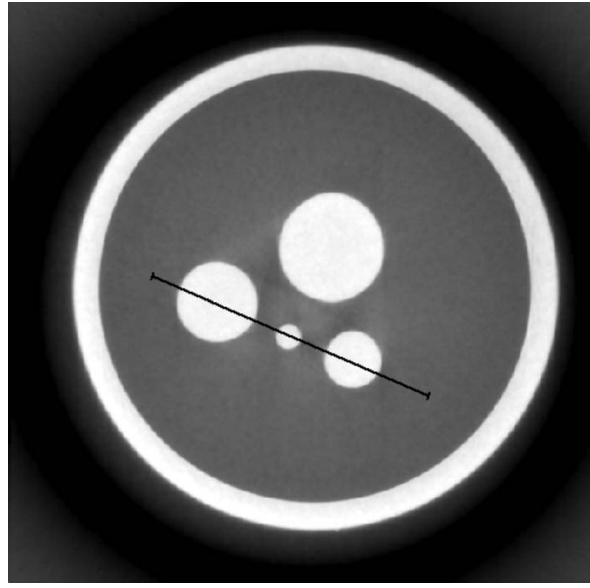

FIG. 2. Axial slice through the reconstructed distribution of the imaginary part of the refractive index in the phantom; straight black line shows the position of a cross section.

intensity, $P_{M}(\mathbf{z})=P_{\text {source }}[\mathbf{z} /(1-M)] * P_{\text {detector }}(\mathbf{z})$ is the normalized PSF of the imaging system which accounts for both the finite size of the source and the spatial resolution of the detector [29], and “*” denotes two-dimensional convolution. Equation (5) is our central result which represents a polychromatic cone-beam PCT reconstruction formula that allows reconstruction of a three-dimentional (3D) distribution of the spectrally averaged refractive index in a nonabsorbing object from a single polychromatic projection image acquired at each view angle. Note that unlike an equivalent reconstruction formula in conventional CT [2], Eq. (5) is stable with respect to high-frequency noise due to the suppression of the growth of the ramp filter, $\left|\xi_{1}\right|$, by the "phase retrieval" kernel $\xi^{2}$ in the denominator $[11,18,30]$. Note also that when a scaling law of the type $\Delta(\mathbf{x}, \lambda)=f\left(\lambda, \lambda_{0}\right) \Delta\left(\mathbf{x}, \lambda_{0}\right) \quad$ is applicable $\quad$ e.g., $\Delta(\mathbf{x}, \lambda)$ $=\left(\lambda / \lambda_{0}\right)^{2} \Delta\left(\mathbf{x}, \lambda_{0}\right)$ as typically holds outside $\mathrm{x}$-ray absorption edges of constituent materials], the true 3D distribution of the refractive index can be recovered from the spectrally averaged refractive index according to $\Delta\left(\mathbf{x}, \lambda_{0}\right)=C\left(\lambda_{0}\right) \Delta_{S}(\mathbf{x})$, provided the position-independent spectral factor $C\left(\lambda_{0}\right)$ $\equiv I_{\text {in }} / \int_{0}^{\infty} S_{i n}(\lambda) D(\lambda) f\left(\lambda, \lambda_{0}\right) d \lambda$ is known. When any scaling law of the above type holds, but $C\left(\lambda_{0}\right)$ is not known, Eq. (5) still provides a distribution equal to the refractive index $\Delta\left(\mathbf{x}, \lambda_{0}\right)$ up to a multiplicative factor.

Under additional conditions, Eq. (5) can be extended to weak monomorphous objects. Here we have $T(\xi, \lambda)$ $\cong \pi \lambda R^{\prime} \xi^{2}+\varepsilon(\lambda)$, and hence $k T(\xi, \lambda) \cong 2 \pi^{2} R^{\prime} \xi^{2}+k \varepsilon(\lambda)$. We see that while the first additive term (which corresponds to phase contrast) is achromatic, the second term (which corresponds to absorption contrast) is not. It is easy to verify that when $d<\sqrt{\pi \lambda R^{\prime} / \varepsilon(\lambda)}$, the phase contrast term is dominant at all nonzero spatial frequencies. As absorption has been assumed weak, this condition is not unrealistic. For example, in the case of $12 \mathrm{keV} \times$ rays, $\varepsilon(\lambda)$ is typically of the order of $10^{-3}$ or so for most biological materials, therefore the last condition limits the maximum size of the sample to $\sim 0.2 \mathrm{~mm}$ for a defocus distance $R^{\prime}=10 \mathrm{~cm}$. Further, we approximate $\varepsilon(\lambda) / \lambda \cong \varepsilon\left(\lambda_{0}\right) / \lambda_{0}$ within the range

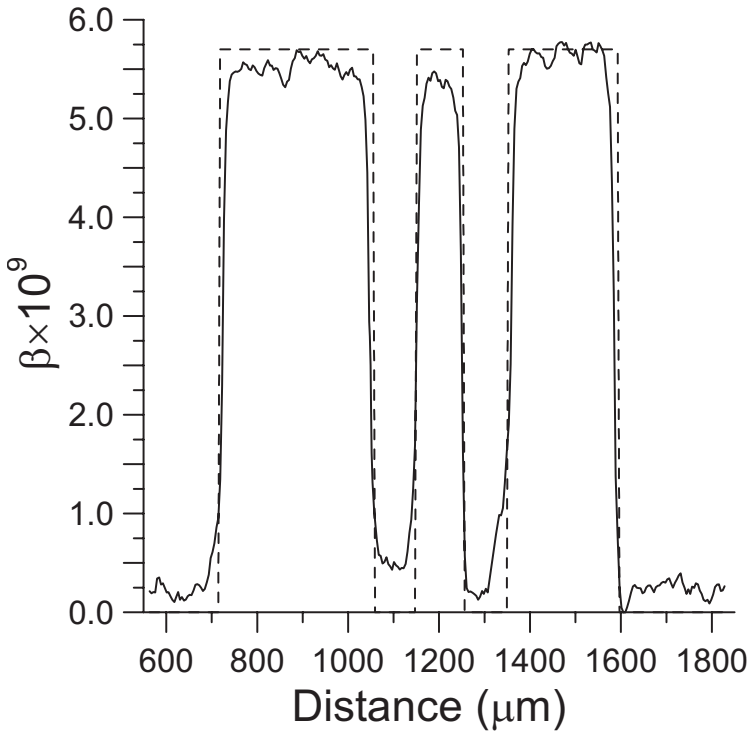

FIG. 3. One-dimensional cross section through the reconstructed distribution of the imaginary part of the refractive index taken along the black line in Fig. 2 (solid line), and the corresponding theoretical cross section (dashed line).

$\left|\lambda-\lambda_{0}\right| / \lambda_{0} \leqslant \sigma(\varepsilon)<1$, where $\sigma(\varepsilon)$ is a monotonic positivevalued function. This implies that $\varepsilon(\lambda) / \lambda$ does not vary too much within that range. Indeed, for example, it is well known that for $\mathrm{x}$-ray wavelengths outside the absorption edges of a sample's component materials, the real and imaginary parts of the refractive index scale as $\beta(\mathbf{x}, \lambda)$ $=\left(\lambda / \lambda_{0}\right)^{4} \beta\left(\mathbf{x}, \lambda_{0}\right)$ and $\Delta(\mathbf{x}, \lambda)=\left(\lambda / \lambda_{0}\right)^{2} \Delta\left(\mathbf{x}, \lambda_{0}\right)$, respectively [3]. Therefore in this case $\left|\varepsilon(\lambda) / \lambda-\varepsilon\left(\lambda_{0}\right) / \lambda_{0}\right|$ $\leqslant \sigma(\varepsilon) \varepsilon\left(\lambda_{0}\right) / \lambda_{0}$ and hence $\varepsilon(\lambda) / \lambda \cong \varepsilon\left(\lambda_{0}\right) / \lambda_{0}$ is a sufficiently good approximation for the relatively insignificant absorption term. A derivation similar to that used above for Eq. (5) now leads to the following reconstruction formula:

$$
\begin{aligned}
\Delta_{S}(\mathbf{x})= & -\frac{R_{1} \rho M^{2}}{8 \pi I_{i n}} \int_{0}^{2 \pi} \frac{1}{p_{2}^{2}} \\
& \times \mathbf{F}_{2}^{-1}\left[\frac{\left|\xi_{1}\right| \mathbf{F}_{2}\left[I_{R_{2}}(M \mathbf{z}, \alpha)\right]}{\left[\pi R^{\prime} \xi^{2}+\varepsilon\left(\lambda_{0}\right) / \lambda_{0}\right] \mathbf{F}_{2}\left[P_{M}(M \mathbf{z})\right]}\right] d \alpha .
\end{aligned}
$$

Equation (6) defines the polychromatic cone-beam PACT algorithm for weakly absorbing monomorphous objects, where the phase-retrieval step is merged with the deconvolution of the PSF and with the filter used in the standard FDK algorithm. This results in a partial cancellation of singularities of the CT filter function, phase-retrieval kernel, and the PSF of the imaging system, in a manner similar to that demonstrated in Ref. [29] in the case of 2D imaging. Furthermore, the weak absorption term acts as a regularizer in the denominator of Eq. (6), increasing the low-frequency stability compared to Eq. (5) (cf. [24]).

We now turn to an experimental implementation of our method. We prepared a phantom consisting of a hollow Perspex tube of $2 \mathrm{~mm}$ external diameter with $100 \mu \mathrm{m}$ thick walls and four different nylon fibers with diameters of 100 , 240,330 , and $420 \mu \mathrm{m}$ inserted parallel to the axis of the 
Perspex tube and fixed in the caps at the top and bottom of the tube. A tomographic dataset was acquired on a pointprojection x-ray ultra microscope (XUM) based on an FEI XL-30 scanning electron microscope [14]. The $\mathrm{x}$ rays were generated by focusing a $30 \mathrm{keV}$ electron beam onto a $500 \mathrm{~nm}$ thick tantalum foil target, with the resulting characteristic radiation and bremsstrahlung yielding a divergent polychromatic X-ray source of approximately $0.2 \mu \mathrm{m}$ diameter, and a mean photon energy of approximately $E_{0}$ $=8.4 \mathrm{keV}$. The presence of characteristic Ta $L$ lines in the spectrum has the effect of making the data effectively more monochromatic, but the contribution of the bremsstrahlung was large enough to prohibit the use of a quasimonochromatic approximation [14]. A total of 720 images of the sample were acquired with equal rotational steps of $0.5^{\circ}$ between the views. Each projection image took $1 \mathrm{~min}$ to acquire, and the total CT scan time was more than $15 \mathrm{~h}$. The source-to-sample distance $R_{1}$ was $25 \mathrm{~mm}$, with a source-todetector distance $R_{1}+R_{2}$ of $259 \mathrm{~mm}$. This geometry gives a geometric magnification of $M=10.4 \times$ at the surface of the detector, producing propagation-based phase-contrast images. Note that the phase contrast present in these projections is an inevitable consequence of the geometric magnification achieved in point-projection x-ray microscopy. The present experimental approach allows one to collect high-resolution images without $\mathrm{x}$-ray focusing elements and without a high- resolution detector. Using the collected projection data, a numerical implementation of Eq. (6) was used for the tomographic reconstruction with $\varepsilon\left(\lambda_{0}\right)=0.0016\left(\lambda_{0}=1.48 \AA\right)$. A sample of the reconstructed distribution of $\beta\left(\mathbf{r}, \lambda_{0}\right)$ $\equiv \varepsilon\left(\lambda_{0}\right) \Delta\left(\mathbf{r}, \lambda_{0}\right)$ is shown in Fig. 2. The spatial resolution of this reconstruction was $3.86 \mu \mathrm{m}$ per cubic voxel. Finally, Fig. 3 shows a cross section of the reconstructed distribution of $\beta\left(\mathbf{r}, \lambda_{0}\right)$ along the black line in Fig. 2 passing through three lower fibers, alongside the ideal theoretical profile of $\beta\left(\mathbf{r}, \lambda_{0}\right)$ at the same wavelength. Note that despite the value of $\varepsilon$ being quite small, the observed x-ray attenuation reached almost 50\% in some projections. It was difficult to avoid significant absorption, as our XUM microscope becomes less efficient at higher $\mathrm{x}$-ray energies. This means that the condition for the dominance of the phase contrast formulated above was violated in the experiment, and as a consequence we did observe some beam hardening in the form of mild "cupping" artifacts. Nevertheless, one can see from Figs. 2 and 3 that a reasonable quantitative accuracy has been achieved in the reconstruction using the CT data collected with an unfiltered broad-band cone beam of $\mathrm{x}$ rays.

The authors are grateful to Dr. A. Pogany and Dr. Ya.I. Nesterets for helpful discussions. D.M.P. acknowledges funding from the Australian Research Council.
[1] A. C. Kak and M. Slaney, Principles of Computerized Tomographic Imaging (SIAM, Philadelphia, 2001).

[2] F. Natterer, The Mathematics of Computerized Tomography (Wiley, New York, 1986).

[3] R. Fitzgerald, Phys. Today 53(7), 23 (2000).

[4] J. Řeháček, Z. Hradil, J. Pekina, S. Pascazio, P. Facchi, and M. Zawisky, Adv. Imaging Electron Phys. 142, 53 (2006).

[5] E. Wolf, Opt. Commun. 1, 153 (1969).

[6] A. J. Devaney, Inverse Probl. 2, 161 (1986).

[7] A. Momose, T. Takeda, and Y. Itai, Rev. Sci. Instrum. 66, 1434 (1995).

[8] U. Bonse and F. Busch, Prog. Biophys. Mol. Biol. 65, 133 (1996).

[9] C. Raven, A. Snigirev, I. Snigireva, P. Spanne, A. Souvorov, and V. Kohn, Appl. Phys. Lett. 69, 1826 (1996).

[10] P. Cloetens, W. Ludwig, J. Baruchel, D. Van Dyck, J. Van Landuyt, J. P. Guigay, and M. Schlenker, Appl. Phys. Lett. 75, 2912 (1999).

[11] A. V. Bronnikov, Opt. Commun. 171, 239 (1999).

[12] F. A. Dilmanian, Z. Zhong, B. Ren, X. Y. Wu, L. D. Chapman, I. Orion, and W. C. Thomlinson, Phys. Med. Biol. 45, 933 (2000)

[13] K. M. Pavlov, C. M. Kewish, J. R. Davis, and M. J. Morgan, J. Phys. D 34, A168 (2001).

[14] S. C. Mayo, T. J. Davis, T. E. Gureyev, P. R. Miller, D. Paganin, A. Pogany, A. W. Stevenson, and S. W. Wilkins, Opt. Express 11, 2289 (2003).

[15] M. A. Anastasio, D. Shi, Y. Huang, and G. Gbur, J. Opt. Soc. Am. A 22, 2651 (2005).
[16] X. Wu and H. Liu, Opt. Express 13, 6000 (2005).

[17] A. Momose, W. Yashiro, Y. Takeda, Y. Suzuki, and T. Hattori, Jpn. J. Appl. Phys., Part 1 45, 5254 (2006).

[18] T. E. Gureyev, D. M. Paganin, G. R. Myers, Ya. I. Nesterets, and S. W. Wilkins, Appl. Phys. Lett. 89, 034102 (2006).

[19] F. Pfeiffer, C. Kottler, O. Bunk, and C. David, Phys. Rev. Lett. 98, 108105 (2007).

[20] M. Engelhardt, J. Baumann, M. Schuster, C. Kottler, F. Pfeiffer, O. Bunk, and C. David, Appl. Phys. Lett. 90, 224101 (2007).

[21] D. M. Paganin, Coherent X-Ray Optics (Oxford University Press, New York, 2006).

[22] S. W. Wilkins, T. E. Gureyev, D. Gao, A. Pogany, and A. W. Stevenson, Nature (London) 384, 335 (1996).

[23] L. D. Turner, K. P. Weber, D. Paganin, and R. E. Scholten, Opt. Lett. 29, 232 (2004).

[24] D. Paganin, S. C. Mayo, T. E. Gureyev, P. R. Miller, and S. W. Wilkins, J. Microsc. 206, 33 (2002).

[25] L. D. Turner, K. F. E. M. Domen, and R. E. Scholten, Phys. Rev. A 72, 031403(R) (2005).

[26] L. A. Feldkamp, L. C. Davis, and J. W. Kress, J. Opt. Soc. Am. A 1, 612 (1984).

[27] X. Yan and R. M. Leahy, IEEE Trans. Med. Imaging 10, 462 (1991).

[28] J.-P. Guigay, Optik (Stuttgart) 49, 121 (1977).

[29] T. E. Gureyev, Ya. I. Nesterets, D. M. Paganin, A. Pogany, and S. W. Wilkins, Opt. Commun. 259, 569 (2006).

[30] G. R. Myers, T. E. Gureyev, and D. M. Paganin, J. Opt. Soc. Am. A 24, 2516 (2007). 